\title{
Velocity shear and current driven instability in a collisional F-region
}

\author{
P. J. G. Perron ${ }^{1}$, J.-M. A. Noël ${ }^{1}$, and J.-P. St.-Maurice ${ }^{2}$ \\ ${ }^{1}$ Department of Physics, Royal Military College of Canada, Kingston, Ontario, Canada \\ ${ }^{2}$ Department of Physics and Engineering Physics, University of Saskatchewan, Saskatoon, Saskatchewan, Canada
}

Received: 22 May 2008 - Revised: 14 November 2008 - Accepted: 16 December 2008 - Published: 23 January 2009

\begin{abstract}
We have studied how the presence of collisions affects the behavior of instabilities triggered by a combination of shears and parallel currents in the ionosphere under a variety of ion to electron temperature ratios. To this goal we have numerically solved a kinetic dispersion relation, using a relaxation model to describe the effects of ion and electron collisions. We have compared our solutions to expressions derived in a fluid limit which applied only to large electron to ion temperature ratios. We have limited our study to threshold conditions for the current density and the shears. We have studied how the threshold varies as a function of the wave-vector angle direction and as a function of frequency. As expected, we have found that for low frequencies and/or elevated ion to electron temperature ratios, the kinetic dispersion relation has to be used to evaluate the threshold conditions. We have also found that ion velocity shears can significantly lower the field-aligned threshold current needed to trigger the instability, especially for wave-vectors close to the perpendicular to the magnetic field. However the current density and shear requirements remain significantly higher than if collisions are neglected. Therefore, for ionospheric F-region applications, the effect of collisions should be included in the calculation of instabilities associated with horizontal shears in the vertical flow. Furthermore, in many situations of interest the kinetic solutions should be used instead of the fluid limit, in spite of the fact that the latter can be shown to produce qualitatively valid solutions.
\end{abstract}

Keywords. Magnetospheric physics (Plasma waves and instabilities) - Space plasma physics (Kinetic and MHD theory; Waves and instabilities)

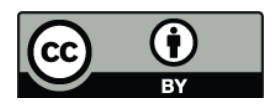

Correspondence to: J.-M. A. Noël (jean-marc.noel@rmc.ca)

\section{Introduction}

As the spatial resolution of ionospheric and magnetospheric measurements has increased over time to reveal sharp density and velocity structures, there has been considerable interest in recent years in studying the possibility of destabilizing space plasmas with velocity shears. There has, in particular, been both direct and indirect evidence for sharp horizontal structures in field-aligned currents (Maggs and Davis, 1968; Marklund et al., 1982; Cerisier et al., 1987; Berthelier et al., 1988; Earle et al., 1989; Trondsen and Cogger, 1997; Noël, 1999; Noël et al., 2000, 2005; Rother et al., 2007). Similarly, field-aligned ion drifts of the order of $\mathrm{km} / \mathrm{s}$, have also been uncovered over narrow horizontal ranges at high latitudes (Liu and Lu, 2004; Ganguli et al., 1994). The two phenomena, intense bursts of field-aligned currents, and large localized ion upflows, may in fact be colocated since type2 thermal ion upflows (TIU) have been shown to be on the edge of auroral arcs, where narrow but intense parallel current densities also exist (e.g. Wahlund et al., 1992; Forme, 1999). The collocation may well not be accidental since a field-aligned electric field and the presence of some remnant high frequency turbulence seems to be the only way to enhance the vertical bulk motion of the ions (Kagan and St.Maurice, 2005).

The kinds of observations that we just described have created a renewed interest in the role played by horizontal shears in field-aligned flows in the excitation of plasma waves in ionospheric and magnetospheric plasmas. Thus, in an earlier theoretical study on the subject, following an original idea by D'Angelo (1965), Basu and Coppi (1989) suggested, using fluid theory, that in the presence of collisions, horizontal shears in the field-aligned ion velocity could produce very low frequency modes in the frame of reference of a moving F-region plasma at an angle very close to perpendicularity to the magnetic field (by low frequency we mean here $\omega \ll k C_{\text {s }}$, where $\omega$ is the frequency of the wave in the moving frame, $k$ is the wave-number, and $C_{\mathrm{s}}$ is the ion-acoustic speed).

Published by Copernicus Publications on behalf of the European Geosciences Union. 
Later, Gavrishchaka et al. (1998) generalized the Basu and Coppi (1989) work to higher frequencies and arbitrary angles of the wave-vector to the magnetic field. However, they neglected collisions in the process. Nevertheless, they found that, in the absence of collisions and finite Larmor radius corrections, they could rather easily excite ion-acoustic waves in the presence of horizontal shears in vertical plasma drifts. Overall, they concluded that both the current-driven electrostatic ion-acoustic mode (CDEIA) and the current driven electrostatic ion cyclotron mode (CDEIC) could be excited with parallel drifts significantly below the critical drift for the homogenous CDEIC and CDEIA. They also found that the threshold current was insensitive to the temperature ratio $\tau=T_{\mathrm{i}} / T_{\mathrm{e}}$ and furthermore concluded that infinitesimal shears could destabilize waves.

The Gavrishchaka et al. (1998) work was later extended in a series of papers. Gavrishchaka et al. (1999) explored the weak and strong shear limits of the earlier paper to conclude that for weak shears the minimum field-aligned currents were indeed much smaller than the critical current for both the CDEIA and CDEIC instabilities. For the strong shear limit, no field aligned current was required at all to destabilize ion-acoustic modes. This work was then extended by Scime et al. (2002) to include thermal anisotropy and by Spangler et al. (2002) who considered the effect of ion and electron temperature anisotropies. None of these extensions of the original study, however, included collisions, which raises the issue of their applicability to ionospheric situations. To this goal, St.-Maurice et al. (2006) provided an extension of the original Gavrishchaka et al. (1998) work in which they added collisions. However, the dispersion relation contained so many parameters that they limited their study to a quasi-fluid limit that considered large ion arguments and small electron arguments. This limit, which contained both collisional and finite Larmor radius effects, was then compared to previous studies, from the initial study of Kindel and Kennel (1971) to the current convective work of Ossakow and Chaturvedi (1979), the work of Basu and Coppi (1989) and of course the results of Gavrishchaka et al. (1998). Within the fluid limit (requiring, in particular, that $T_{\mathrm{e}} \gg T_{\mathrm{i}}$ ), it was found that collisions and Larmor radius corrections both acted to modify the threshold conditions in the regimes explored by Basu and Coppi (1989) and Gavrishchaka et al. (1998). In particular, collisions usually meant that the plasma could no longer become unstable to infinitesimal shears. In addition, St.-Maurice et al. (2006) found a new mode with a cyclotron flavor at intermediate aspect angles. In terms of specific numbers, overall, in the absence of shears they got ion acoustic modes for vertical drifts of the order of $10-100 C_{\mathrm{s}}$, which were similar to the numbers obtained by Kindel and Kennel (1971). However, shears always introduced a near zero current mode for $\omega / k C_{\mathrm{s}}<1$; for moderate shears $\left(S_{\mathrm{i}}=V_{\mathrm{di}}^{\prime} / \Omega_{\mathrm{i}}<0.1\right)$, the generalization looked similar to the Basu and Coppi (1989) expression with modes at a small fraction of $k C_{\mathrm{s}}$.
In the present paper, we want to continue to explore the St.-Maurice et al. (2006) solutions by solving the full kinetic dispersion relation. We use their fluid solutions as a starting point because they can be and have been studied in detail in terms of physical processes that could be tracked; this type of study is not so easy to do with the kinetic solution. As our starting point, we therefore compare fluid and kinetic solutions under moderate drifts and large electron temperatures $T_{\mathrm{e}}$. We then proceed to systematically move away from these restrictions and we study how the threshold changes. Using this type of approach allows us to keep track of the evolution of a mode by beginning with its quasi-fluid behavior while at the same time tracking more easily its physical origin.

Our paper is organized as follows: in Sect. 2 we present the generalized dispersion relation derived by St.-Maurice et al. (2006). In Sect. 3 we compare the results using the fluidlike dispersion relation presented previously by St.-Maurice et al. (2006) with those obtained using the general kinetic dispersion relation. Finally, we explore the kinetic dispersion relation for modes that can be excited by vertical ion drifts under appropriate F-region conditions.

\section{The generalized kinetic dispersion relation}

A kinetic dispersion relation for electrostatic current and shear driven instabilities in a collisional plasma has been presented by St.-Maurice et al. (2006). The model assumed that there was a vertical drift in the thermal electrons and/or ions and that the drift had a horizontal gradient in the $\mathrm{x}$-direction (i.e. a shear) in the plane perpendicular to the magnetic field. In this paper, we only present the generalized kinetic dispersion relation itself and refer the reader to St.-Maurice et al. (2006) for the details of the derivation.

The St.-Maurice et al. (2006) dispersion relation for electrostatic modes is written as follows

$$
\frac{H_{\mathrm{i}}(\mathbf{k}, \omega)}{1+U_{\mathrm{i}}(\mathbf{k}, \omega)}+\frac{H_{\mathrm{e}}(\mathbf{k}, \omega)}{1+U_{\mathrm{e}}(\mathbf{k}, \omega)}+k^{2} \lambda_{\mathrm{di}}^{2}=0
$$

where the $H_{\mathrm{j}}(\mathbf{k}, \omega)$ function is known as the $\mathrm{j}^{\text {th }}$ component of the susceptibility, $k$ is the wavenumber and $\lambda_{\text {di }}$ is the Debye length for the ions.

The expression for the susceptiblity of the ions can be written as follows (Perron, 2004; St.-Maurice et al., 2006)

$$
\begin{aligned}
& H_{\mathrm{i}}=1-\frac{k_{y} V_{\mathrm{di}}^{\prime}}{\left|k_{z}\right| \Omega_{\mathrm{i}}}+\sum_{n} \Gamma_{n}\left(k_{y}^{2} \rho_{\mathrm{i}}^{2}\right)\left(\frac{\varpi+i v_{\mathrm{i}}}{\sqrt{2}\left|k_{z}\right| v_{\mathrm{ti}}}\right) Z\left(\frac{\varpi+i v_{\mathrm{i}}-n \Omega_{\mathrm{i}}}{\sqrt{2}\left|k_{z}\right| v_{\mathrm{ti}}}\right) \\
& -\sum_{n} \Gamma_{n}^{*}\left(k_{y}^{2} \rho_{\mathrm{i}}^{2}\right) \frac{k_{y} V_{\mathrm{di}}^{\prime}}{\left|k_{z}\right| \Omega_{\mathrm{i}}}\left(\frac{\varpi+i v_{\mathrm{i}}-n \Omega_{\mathrm{i}}}{\sqrt{2}\left|k_{z}\right| v_{\mathrm{ti}}}\right) Z\left(\frac{\varpi+i v_{\mathrm{i}}-n \Omega_{\mathrm{i}}}{\sqrt{2}\left|k_{z}\right| v_{\mathrm{ti}}}\right)
\end{aligned}
$$


while the susceptibility for the electrons can be written as

$$
\begin{aligned}
& H_{\mathrm{e}}=\frac{T_{\mathrm{i}}}{T_{\mathrm{e}}}\left[1+\left(\frac{\omega-k_{z} V_{\mathrm{de}}+i v_{\mathrm{e}}}{\sqrt{2}\left|k_{z}\right| v_{\mathrm{te}}}\right) Z\left(\frac{\omega-k_{z} V_{d e}+i v_{\mathrm{e}}}{\sqrt{2}\left|k_{z}\right| v_{\mathrm{te}}}\right)\right. \\
& \left.+\frac{k_{y} V_{\mathrm{de}}^{\prime}}{\left|k_{z}\right| \Omega_{\mathrm{e}}}\left\{1+\left(\frac{\omega-k_{z} V_{\mathrm{de}}+i v_{\mathrm{e}}}{\sqrt{2}\left|k_{z}\right| v_{\mathrm{te}}}\right) Z\left(\frac{\omega-k_{z} V_{\mathrm{de}}+i v_{\mathrm{e}}}{\sqrt{2}\left|k_{z}\right| v_{\mathrm{te}}}\right)\right\}\right]
\end{aligned}
$$

where $\varpi=\omega-k_{z} V_{\mathrm{di}}, V_{\mathrm{di}, \mathrm{e}}^{\prime}=d V_{\mathrm{di}, \mathrm{e}} / d x$ is the ion/electron velocity shear, $\lambda_{\mathrm{di}}$ is the Debye length for the ions, $\Omega_{\mathrm{i}, \mathrm{e}}$ is the ion, electron gyrofrequency, $V_{\mathrm{di}, \mathrm{e}}$ is the ion/electron drift velocity along the magnetic field line and $v_{\mathrm{ti}, \mathrm{e}}=\sqrt{T_{\mathrm{i}, \mathrm{e}} / m_{\mathrm{i}, \mathrm{e}}}$ is the thermal velocity of the ions/electrons (using $\mathrm{eV}$ units for $\left.T_{\mathrm{i}, \mathrm{e}}\right), v_{\mathrm{i}, \mathrm{e}}$ is the ion/electron momentum transfer collision frequency and, $\rho_{\mathrm{i}}=\sqrt{T_{\mathrm{i}} / m_{\mathrm{i}} \Omega_{\mathrm{i}}^{2}}$ is the Larmor radius for the ions. To get the last term for $H_{\mathrm{e}}$ in Eq. (3) we have used the low frequency limit $\omega \ll \Omega_{\mathrm{e}}$ or equivalently $\rho_{\mathrm{e}}^{2} \rightarrow 0$.

The function $\Gamma_{n}(b)$ is given by

$$
\Gamma_{n}(b)=I_{n}(b) e^{-b}
$$

where $I_{n}(b)$ is the modified Bessel function. A similar contribution is associated with the shear term $V_{\mathrm{di}}^{\prime}$ in Eq. (2) in association with $\Gamma_{n}^{*}$. In comparison with Eq. (4), $\Gamma_{n}^{*}$ contains a finite Larmor radius correction. It is given by

$\Gamma_{n}^{*}(b)=\left[(1-b) I_{n}(b)+b I_{n}^{\prime}(b)\right] e^{-b}$

where $b=k_{y}^{2} \rho_{\mathrm{L}}^{2}$ and $\rho_{\mathrm{L}}=\sqrt{T / m \Omega^{2}}$ is the Larmor radius.

The functions $U_{\mathrm{j}}(\mathbf{k}, \omega)$ in Eq. (1) take the form (see Perron, 2004; St.-Maurice et al., 2006)

$$
\begin{aligned}
U_{\mathrm{i}} & =\frac{i v_{\mathrm{i}}}{\sqrt{2}\left|k_{z}\right| v_{\mathrm{ti}}} \sum_{n} \Gamma_{n}\left(k_{y}^{2} \rho_{\mathrm{i}}^{2}\right) Z\left(\frac{\varpi+i v_{\mathrm{i}}-n \Omega_{\mathrm{i}}}{\sqrt{2}\left|k_{z}\right| v_{\mathrm{ti}}}\right) \\
U_{\mathrm{e}} & =\frac{i v_{\mathrm{e}}}{\sqrt{2}\left|k_{z}\right| v_{\mathrm{te}}} Z\left(\frac{\omega-k_{z} V_{\mathrm{de}}+i v_{\mathrm{e}}}{\sqrt{2}\left|k_{z}\right| v_{\mathrm{te}}}\right)
\end{aligned}
$$

Equations (2-3) and (6-7) can be now put into Eq. (1) and the roots of the resulting dispersion relation can be studied. In their study, St.-Maurice et al. (2006) limited themselves to fluid-like mode solutions by considering large ion and small electron arguments only in Eq. (1). In the present paper, we will study the solutions for the full kinetic solution threshold conditions, i.e., we seek solutions for which $\varpi$ is strictly real $\left(\varpi=\omega_{\mathrm{R}}\right)$. We proceed as in St.-Maurice et al. (2006) by assuming that $\omega_{\mathrm{R}} / k C_{\mathrm{S}}$ and $\Omega_{\mathrm{i}} / k C_{\mathrm{s}}$ are given and we solve Eq. (1) for what the shear and drift parameter $\left(S_{\mathrm{i}}=V_{\mathrm{di}}^{\prime} / \Omega_{\mathrm{i}}=k_{z} \zeta_{\mathrm{i}} / k_{y}\right)$ and $\left(\Delta V_{\mathrm{d}} / C_{\mathrm{s}}=\left(V_{\mathrm{de}}-V_{\mathrm{di}}\right) / C_{\mathrm{s}}\right)$ need to be for different values of the angle $\theta$ between the wavevector and the magnetic field.

\section{Results}

In this section we study the threshold conditions for instability using the full kinetic dispersion relation Eq. (1) for a variety of input parameters. We start by comparing the results obtained from the fluid dispersion relation (presented in St.-Maurice et al., 2006) with those obtained using Eq. (1). We show that under the appropriate input parameters, the quasi-fluid results are identical to those obtained from the kinetic dispersion relation. This requires large enough values of $\omega_{\mathrm{R}} / k C_{\mathrm{s}}$.

For the second part of our study, we examine how the temperature ratio $T_{\mathrm{i}} / T_{\mathrm{e}}$ affects the threshold conditions. To that goal we use the generalized kinetic dispersion relation so as to be able to increase the ratio to values of order 1 , which is outside the range for which the quasi-fluid expressions are valid.

The final part of our study is concerned with the effects of collisions on the threshold conditions. For temperature ratios of order 1, we examine the effect on the solution of a change in the electron to ion collision frequency ratio as well as the effect of the ion to cyclotron frequency ratio. This allows for a study of different possible ionospheric regimes.

\subsection{Comparing the fluid dispersion relation and the generalized kinetic dispersion relation under small $T_{\mathbf{i}} / T_{\mathrm{e}}$ ratios}

The quasi-fluid expressions that were presented in St.Maurice et al. (2006) were based on large ion arguments and small electron arguments, which is equivalent, basically, to having a small $T_{\mathrm{i}} / T_{\mathrm{e}}$ ratio. We therefore start our exploration with a comparison between the quasi-fluid solution and the kinetic results under vanishingly small values of $T_{\mathrm{i}} / T_{\mathrm{e}}$. This has the advantage of grounding our study on the somewhat simpler (and easier to understand) fluid solutions, before embarking into more general cases that cannot directly be compared to fluid modes.

In the upper panel of Fig. 1, we present the threshold conditions as a function of the magnitude of the relative drift $\left|\Delta V_{\mathrm{d}} / C_{\mathrm{s}}=\left(V_{\mathrm{di}}-V_{\mathrm{de}}\right) / C_{\mathrm{s}}\right|$ and of the ion shear $S_{\mathrm{i}}=V_{\mathrm{di}}^{\prime} / \Omega_{\mathrm{i}}$. The results are computed using Eqs. (A1-A5) of St.-Maurice et al. (2006). We use as input $\Omega_{\mathrm{i}} / k C_{\mathrm{s}}=2.0, v_{\mathrm{e}} / \nu_{\mathrm{i}}=10.0$, $\nu_{\mathrm{i}} / \Omega_{\mathrm{i}}=0.01$ and $T_{\mathrm{i}} / T_{\mathrm{e}}=10^{-6}$. Each curve represents the solution or threshold values $\left(\Delta V_{\mathrm{d}} / C_{\mathrm{s}}, S_{\mathrm{i}}\right)$ as the angle, $\theta=\arctan \left(k_{z} / k_{y}\right)$ is swept from $0^{\circ}$ to $90^{\circ}$ for $\omega_{\mathrm{R}} / k C_{\mathrm{s}}=0.02$, $0.1,0.5,0.9$ and 1.01. The $y$-axis for both panels in Fig. 1 represents the threshold values of the ion shear, $S_{\mathrm{i}}$, while the $\mathrm{x}$-axis represents the corresponding values of the relative drift, $\Delta V_{\mathrm{d}} / C_{\mathrm{s}}$. Each point on the solution curves corresponds to a specific angle $\theta$, between the wavevector and the magnetic field.

The results presented in the upper panel of Fig. 1 are identical to those presented in St.-Maurice et al. (2006) (their Fig. 4) except for the trace corresponding to the lowest value of $\omega_{\mathrm{R}} / k C_{\mathrm{S}}$ namely, $\omega_{\mathrm{R}} / k C_{\mathrm{S}}=0.02$. When the complete fluid dispersion relation is used, as is the case here, we observe the existence of an additional solution branch for near zero shears and relative drifts between $\sim 1.5 C_{\mathrm{s}}$ and $\sim 1000 C_{\mathrm{S}}$ 

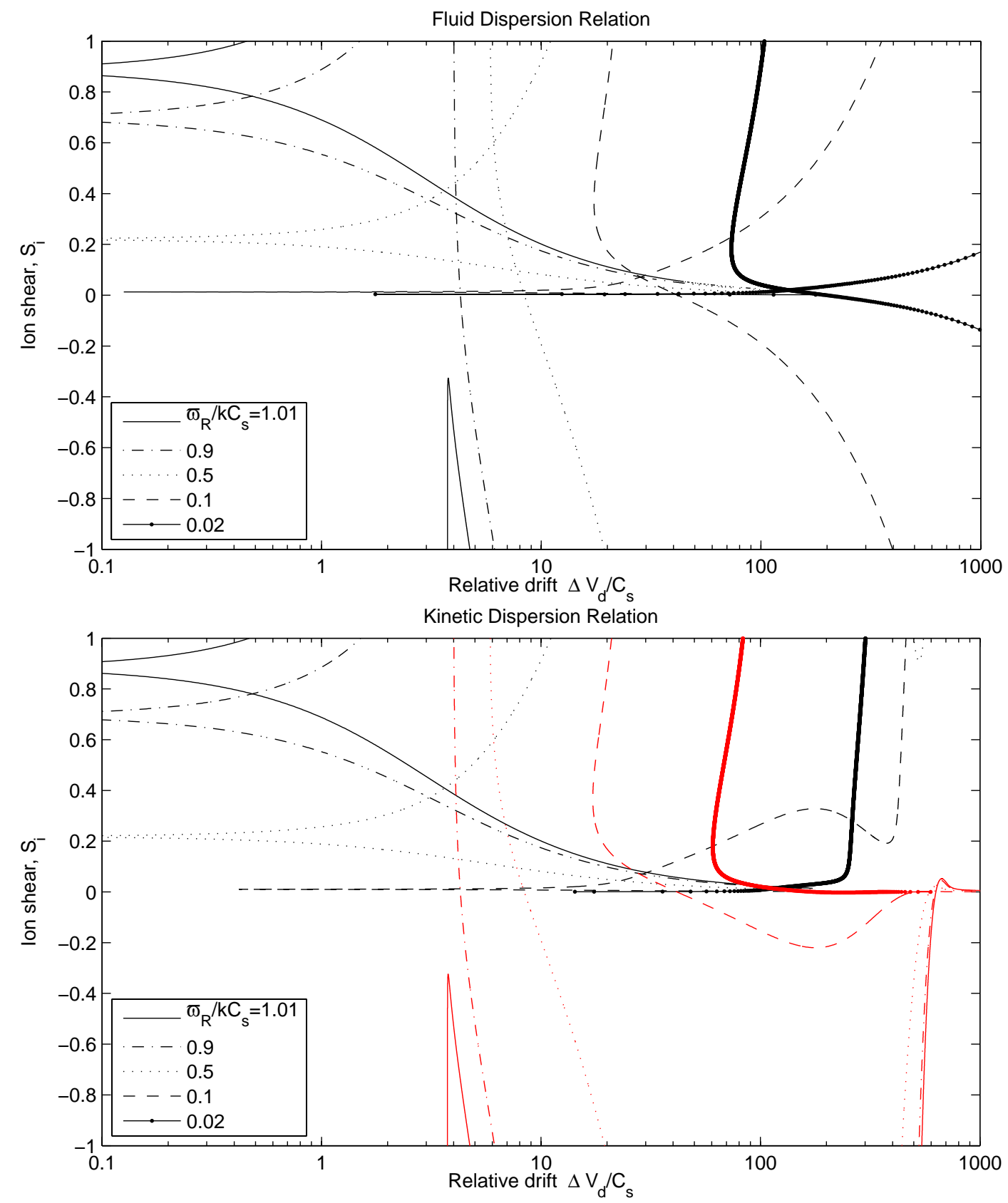

Fig. 1. Threshold conditions obtained from the fluid-like dispersion relation using Eqs. (A1-A5) of St.-Maurice et al. (2006) (top panel) and using the full kinetic dispersion relation Eq. (1) (lower panel). Results are presented for $\omega_{\mathrm{R}} / k C_{\mathrm{S}}=0.02$ (dot-line), 0.1 (dashed line), 0.5 (dotted line), 0.9 (dash-dotted line) and 1.01 (solid line) for the collisional case using $\Omega_{\mathrm{i}} /\left(k C_{\mathrm{S}}\right)=2, T_{\mathrm{i}} / T_{\mathrm{e}}=10^{-6}, v_{\mathrm{i}} / \Omega_{\mathrm{i}}=0.01$ and $v_{\mathrm{e}} / \nu_{\mathrm{i}}=10.0$. Red curves in the bottom panel are solutions for which the relative drift never changes signs (crosses zero) as the wave-vector angles are swept from 0 to 90 degrees.

and a shear variation, $S_{\mathrm{i}}$, varying between 0 and 0.19 . The change in the low frequency solution comes from a contribution from the relative drift, through $\Delta V_{\mathrm{d}} / C_{\mathrm{s}}$, in Eq. (A.2) in St.-Maurice et al. (2006). In their study, St.-Maurice et al. (2006) had neglected this term when computing the threshold conditions. In the present study, we have retained all terms, including the normally small $\Delta V_{\mathrm{d}} / C_{\mathrm{s}}$ contribution. Clearly, this contribution is indeed only present at the lowest frequencies. This is evident from the fact that at higher frequencies, all curves are identical with and without the additional $\Delta V_{\mathrm{d}} / C_{\mathrm{s}}$ term. Altogether, this agrees, in the end, with a statement by St.-Maurice et al. (2006) who wrote that it 
would be best to solve the full fluid dispersion relation when $\omega_{\mathrm{R}}<0.1 k C_{\mathrm{s}}$.

The lower panel of Fig. 1 shows plots of the threshold conditions that were obtained using the generalized kinetic dispersion relation Eq. (1) for the same input parameters used with the fluid dispersion relation in the upper panel. The red curves shown in the lower panel of Fig. 1 come from threshold conditions for which the relative drift never changes sign (with $\Delta V_{\mathrm{d}}>0$ in all cases). As a result, no deep minimum can be seen in the magnitude of relative drift on our logarithmic scale, since the relative drift does not go through zero (see the red curves in the lower panel of Fig. 2). The red traces correspond to what we will call the "upper branch" solutions of the kinetic dispersion relation. The importance of the existence of this type of solutions is discussed in more detail later below.

By comparing the two panels in Fig. 1, we observe that for large values of $\omega_{\mathrm{R}} / k C_{\mathrm{s}}$ the kinetic and fluid dispersion relations give identical results for the threshold conditions. The only exceptions are the traces corresponding to the two lowest values of $\omega_{\mathrm{R}} / k C_{\mathrm{s}}$, namely, $\omega_{\mathrm{R}} / k C_{\mathrm{s}}=0.02$ and 0.1 when the relative $\Delta V_{\mathrm{d}} / C_{\mathrm{s}}$ is larger than $\sim 50$. However, this represents a high drift (large current density) regime, which is of less interest to the present work.

We can get another view of the low frequency differences between the solutions from the full fluid dispersion relation used here and the low $\Delta V_{\mathrm{d}}$ approximation solution used in St.-Maurice et al. (2006) by comparing the upper panel of Fig. 2 with their Fig. 5, both of which show how the magnitude of the relative drift changes as a function of the wavevector angle $\theta$. Notice that once again, our present fluid results are shown in the upper panel of Fig. 2 while the kinetic results are shown in the lower panel.

When the upper panel of Fig. 2 is compared to Fig. 5 of St.-Maurice et al. (2006) the most important difference is the existence of a localized minimum in the relative drift for $\omega_{\mathrm{R}} / k C_{\mathrm{s}}=0.02$ at $\theta \sim 0.09^{\circ}$ when the $\Delta V_{\mathrm{d}}$ term is retained in the computation. The localized minimum in the trace for $\omega_{\mathrm{R}} / k C_{\mathrm{S}}=0.02$ in our computation does not appear in Fig. 5 of St.-Maurice et al. (2006). However, all the other curves, which correspond to larger values of $\omega_{\mathrm{R}} / k C_{\mathrm{S}}$, are in very good agreement with those from St.-Maurice et al. (2006).

It should be noted that at the angle where the magnitude of the relative drift is a minimum (specifically, $0.09^{\circ}$ for the $\omega_{\mathrm{R}} / k C_{\mathrm{s}}=0.02$ case), the relative drift changes sign from positive on the left hand side of the minimum to negative on the right hand side of the minimum. The presence of this minimum continues the trend established at larger frequencies for a minimum with sign reversal, that goes to increasingly small values of $\theta$ as the frequency goes down. In addition to this change of sign through a passage through zero, we notice a cusp in the $\omega_{\mathrm{R}} / k C_{\mathrm{s}}=0.02$ curve near $\sim 1.5^{\circ}$. Again, this cusp extends a similar trend seen at higher frequencies. At that cusp, both the shear and the relative drift change their signs. This time, however, the reversal in the sign of $\Delta V_{\mathrm{d}}$ is not done with a passage through 0 , but rather through a singularity. While this occurs, we note that the shear changes sign from positive to negative, thereby indicating that the origin of the singularity in $\Delta V_{\mathrm{d}}$ is related to the change in sign of the shear.

When considering the lower panel of Fig. 2 we first notice that the kinetic results actually contain two "branches" of solutions to Eq. (1) for each frequency $\omega_{\mathrm{R}} / k C_{\mathrm{s}}$. The existence of the two branches is consistent with results obtained by Perron (2004), who undertook a morphological study of the kinetic solutions by plotting the magnitude of Eq. (1) as a function of shear $\left(S_{\mathrm{i}}\right)$ and relative drift $\left(\Delta V_{\mathrm{d}} / C_{\mathrm{s}}\right)$ for a given wavevector angle $(\theta)$. Perron (2004) showed that for any given angle there were actually two solutions. One of the solutions coincides with a large positive relative drift for which we have coined the term "upper branch", while the second solution coincides with smaller relative drifts which change signs and have been labeled here as the "lower branch" solutions. As stated earlier, in order to better differentiate between the two branches in a graphical sense, we have traced the "upper branches" in red and the "lower branches" in black. This color scheme has been used throughout the paper.

In the lower panel of Fig. 2 we observe, first of all, that for large values of $\omega_{\mathrm{R}} / k C_{\mathrm{s}}$ the positions obtained in the minima of the magnitude of $\Delta V_{\mathrm{d}}$ (sign reversals) using the kinetic dispersion relation are usually in excellent agreement with those found using the fluid dispersion relation (upper panel) as well as with those presented by St.-Maurice et al. (2006) in their Fig. 5. The only exceptions are seen in the traces corresponding to the two lowest frequencies, namely, $\omega_{\mathrm{R}} / k C_{\mathrm{s}}=0.02$ and 0.1 . For both of these traces, the minima have been shifted towards the left, that is, to smaller wave-vector angles for the kinetic case compared to the fluid case. For example, for $\omega_{\mathrm{R}} / k C_{\mathrm{S}}=0.02$, the minimum is at $\sim 0.09^{\circ}$ for the fluid case while it is closer to $0.02^{\circ}$ for the kinetic case. In addition, it should be noted that all of the upper branches, shown in red, correspond to positive drifts, $\Delta V_{\mathrm{d}} / C_{\mathrm{s}}>0$. For the lower branches, shown in black, positive drifts, $\Delta V_{\mathrm{d}} / C_{\mathrm{s}}>0$, are found on the left of the minima while negative drifts, $\Delta V_{\mathrm{d}} / C_{\mathrm{s}}<0$, are found on the right of each absolute values minima shown in Fig. 2. Interestingly enough, the angles at which the black traces (lower branch) intercept the red traces (upper branch) for any given frequency always coincide with the location of the cusp (singularity in $\Delta V_{\mathrm{d}}$ ) that appear in the fluid case. The singularity found in the fluid case is therefore an artifact and is replaced in the kinetic dispersion relation by a crossover between two families of solutions.

We can, in the end, summarize our comparison of solutions under low $T_{\mathrm{i}} / T_{\mathrm{e}}$ conditions as follows: first, we have to be aware of the fact that at low frequencies, there is a non-negligible contribution from $\Delta V_{\mathrm{d}}$ that only matters at the lower frequencies but nevertheless allows the fluid modes to continue to have a sign reversal in $\Delta V_{\mathrm{d}}$ at a well defined angle, which is close to perpendicularity at small 

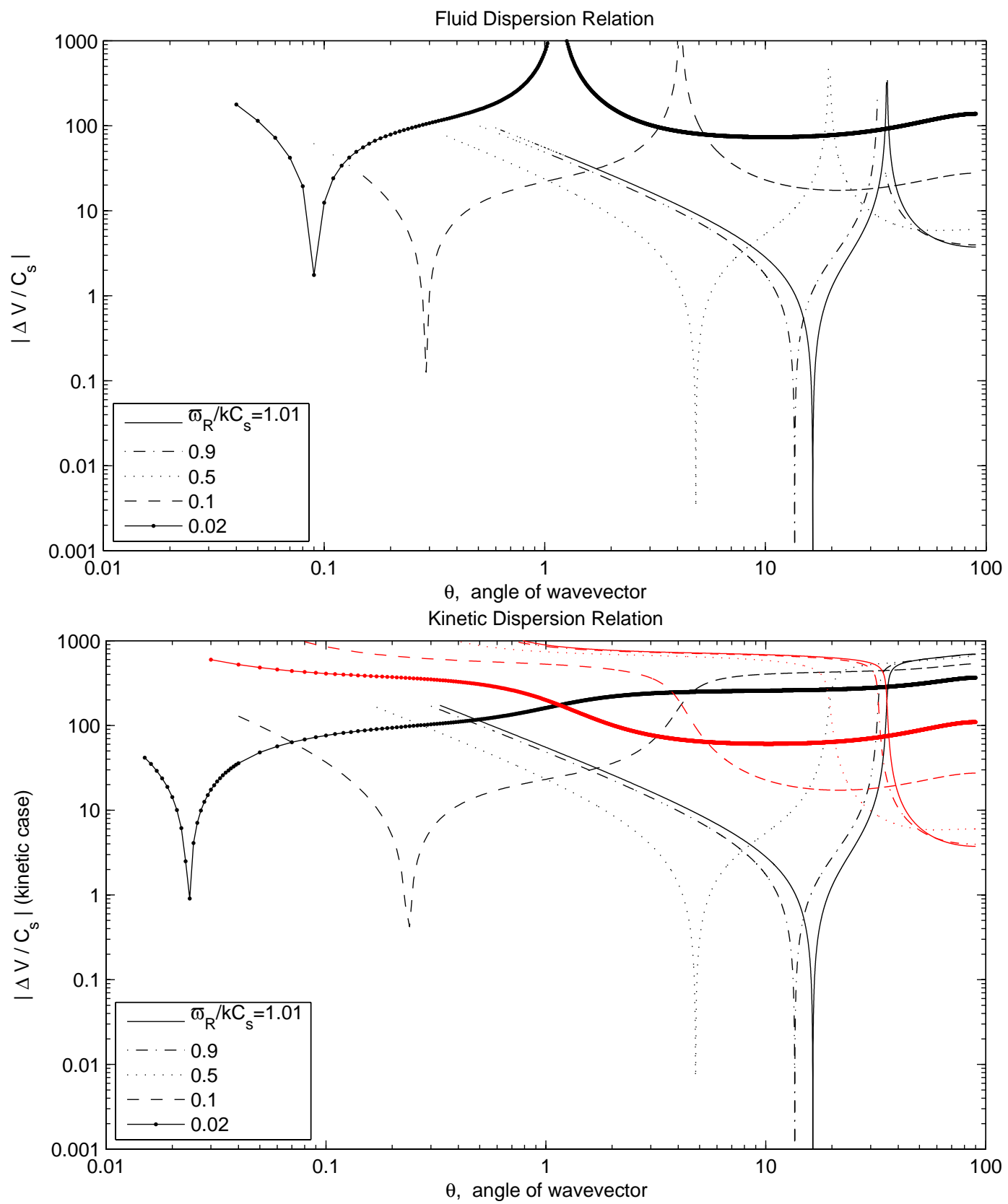

Fig. 2. Relative drift as a function of wavevector angle for the cases described in Fig. 1 . The angle is $0^{\circ}$ when the angle is perpendicular to B and $90^{\circ}$ when parallel to it. The line scheme is the same as in Fig. 1.

frequencies. The small $\Delta V_{\mathrm{d}}$ contribution was not considered by St.-Maurice et al. (2006). Only frequencies such that $\omega_{\mathrm{R}} / k C_{\mathrm{S}}<0.1$ are affected by this correction. Secondly, we find that the kinetic solution has two branches of solutions. The branch that corresponds to smaller values of $\Delta V_{\mathrm{d}}$ and undergoes a sign reversal at a particular wave-vector angle is in excellent agreement with the quasi-fluid solutions in the region of the reversal. One exception occurs, once again at smaller frequencies, where the angle obtained from the ki- netic solution is detectably smaller than the angle from fluid theory (this stated, however, both angles are very close to perpendicularity to the magnetic field anyway). At wavevector angles that are greater than the angle for $\Delta V_{\mathrm{d}}$ reversal, there is a cross-over between the two kinetic solutions. The cross-over occurs at a point where the fluid solutions hit an artificial singularity in $\Delta V_{\mathrm{d}}$. At angles that are beyond the cross over point the fluid solutions move from agreeing with the lower kinetic branch to agreeing with the upper branch 
kinetic solution. However, all these features involve ratios $\left|\Delta V_{\mathrm{d}} / C_{\mathrm{s}}\right|$ of order 100 or more, at least for the situations that we have considered. Since we are interested mostly in threshold associated with relatively low ratios of $\left|\Delta V_{\mathrm{d}} / C_{\mathrm{s}}\right|$, we can conclude that the fluid solutions are valid where it counts the most, namely, in regions where the relative drifts reach their lowest values. At small values of $T_{\mathrm{i}} / T_{\mathrm{e}}$ we can therefore use the fluid results with confidence, at least when the frequency is large enough, which in our case corresponds to $\omega_{\mathrm{R}} / k C_{\mathrm{s}}>0.1$.

\subsection{Kinetic results for larger values of $T_{\mathrm{i}} / T_{\mathrm{e}}$}

In Fig. 3 we present the threshold solutions to Eq. (1) as the temperature ratio $\tau=T_{\mathrm{i}} / T_{\mathrm{e}}$ increases from 0.01 (top panel) to 1.0 (bottom panel). We have used the same input values as in Fig. 1 for the other parameters. As the temperature ratio $\tau$ increases, Fig. 3 clearly shows that the threshold curves begin to deviate significantly from those that were presented in Fig. 1 of the previous section: the red traces or the "upper branch" solutions begin to move towards the right, i.e., towards larger relative drifts for all values of $\omega_{\mathrm{R}} / k C_{\mathrm{s}}$ as $\tau$ increases from 0.01 to 1.0. On the other hand, the black traces or "lower branch" solutions remain relatively stationary while the temperature ratio increases. In that sense, the "lower branch" solutions are relatively insensitive to the temperature ratio $\tau$.

We remind the reader that all of the "upper branch" solutions shown in red in Fig. 3 are associated with strictly positive values of the relative drift $\Delta V_{\mathrm{d}}$. The "lower branch" solutions, shown in black, exhibit increases in $S_{\mathrm{i}}$ with $\left|\Delta V_{\mathrm{d}}\right|$ and are associated with values of $\Delta V_{\mathrm{d}}$ that are relatively small in magnitude and change signs at some value of the angle of the wave-vector.

An easy conclusion that can be drawn from Fig. 3 is that as the temperature ratio increases, the shears required for plasma destabilization can be quite small near zero frequencies while the relative drift requirement can remain relatively modest in these cases. This can be seen by the nearly horizontal black traces for $\omega_{\mathrm{R}}=0.1 k C_{\mathrm{s}}$ and $0.02 k C_{\mathrm{s}}$ near $S_{\mathrm{i}} \approx 0$ for all the temperature ratios presented. In addition, much as was the case for small temperature ratios, the traces in Fig. 3 demonstrate that the threshold requirements on the magnitude of $V_{\mathrm{d}}$ are small to negligible only for a very narrow range of angles $\theta$. This observation is, however, easier to make if we use Fig. 4, which shows how the relative drift changes with the angle of the wavevector. As with Fig. 3, the temperature ratio $\tau$ increases from 0.01 in the top left panel to 1.0 in the lower right panel.

We also observe from Fig. 4 that as $\tau$ increases, the "upper branch" solutions (red traces) systematically move upward towards larger values of the relative drift. The "lower branch" solutions on the other hand, stay relatively stationary with increasing temperature ratios $\tau$. As the black traces in Fig. 4 clearly demonstrate, the threshold condition on the magnitude of the relative drift are only lowered for very narrow ranges of the angle $\theta$ for any given frequency. This is shown in Fig. 4 through deep minima in the traces of the relative drift as a function of angle. The relative drift $\Delta V_{\mathrm{d}}$ is negative on the right side of all the absolute values minima shown in Fig. 4 while $\Delta V_{\mathrm{d}}$ is positive on the left side of the minima.

As $\tau$ increases, the threshold lowering can be considered to be a resonant condition since the angular requirement on the direction of the wavevector is so specific. For example, when $\omega_{\mathrm{R}}=0.9 \mathrm{k} C_{\mathrm{s}}$ the relative drift can be quite modest when the angle is of the order of $15-20^{\circ}$ but the requirement on the shear is that is has to be larger than 1.0 (see Fig. 5). On the other hand, when $\omega_{\mathrm{R}}=0.02 k C_{\mathrm{s}}$ and $\tau=1.0$, the relative drift has a minimum value at $\theta \sim 0.03^{\circ}$ corresponding to a shear on the order of 0.002-0.003 (see bottom right panel of Fig. 5).

As just alluded to, Fig. 5 shows how the shear changes as a function of angle for the near zero $V_{\mathrm{d}}$ cases described in Figs. 3 and 4. In this figure we have only plotted the "lower branch" solutions because the "upper branch" solutions are for much larger relative drifts. The first thing to notice is that the traces do not vary much with $\tau$ except for a small systematic shift of all traces towards the left or equivalently, toward smaller angles as $\tau$ increases. Another point to keep in mind is that, at least for the cases studied here, the plasma is unstable to "small" shears $\left(\left|S_{\mathrm{i}}\right|<0.05\right)$ only when the angle of the wave-vector is within 1 degree of perpendicularity to the magnetic field. We are led to conclude that the most unstable situations when $T_{\mathrm{i}} / T_{\mathrm{e}}$ is greater than 0.1 are those for which the modes are very nearly perpendicular to the magnetic field and approach zero frequency. These results are highly reminiscent of the Basu and Coppi (1989) work. For situations where the wave-vector deviates measurably from perpendicularity while maintaining a small drift requirement, we find that the shears have to be of the order of 0.2. This, however, cannot be considered to be an "infinitesimal" shear. In that case, on the other hand, the frequency could be of order $k C_{\mathrm{s}}$.

\subsection{Effects associated with changing collision frequencies}

We have examined the effect of collisions on threshold conditions using the kinetic dispersion relation Eq. (1). We have first examined the role played by the ratio $\nu_{\mathrm{e}} / \nu_{\mathrm{i}}$ on the threshold conditions. Using the reasonable assumption that the collision frequency in the BGK model is representative of the momentum transfer collision frequency, and using electron-ion collisions and ion-neutral collisions for F-region applications, the ratio $v_{\mathrm{e}} / v_{\mathrm{i}}$ then becomes proportional to $\left(n_{\mathrm{e}} / n_{\mathrm{O}}\right) T_{\mathrm{e}}^{-3 / 2} / \sqrt{\left(T_{\mathrm{i}}+T_{\mathrm{n}}\right)}$ for a background atomic oxygen atmosphere, where $n_{\mathrm{O}}$ is the atomic oxygen density (Schunk and Nagy, 2000). Thus, a study of the effect of $v_{\mathrm{e}} / v_{\mathrm{i}}$ in the Fregion context becomes equivalent to studying the influence of the plasma density and electron temperature. 

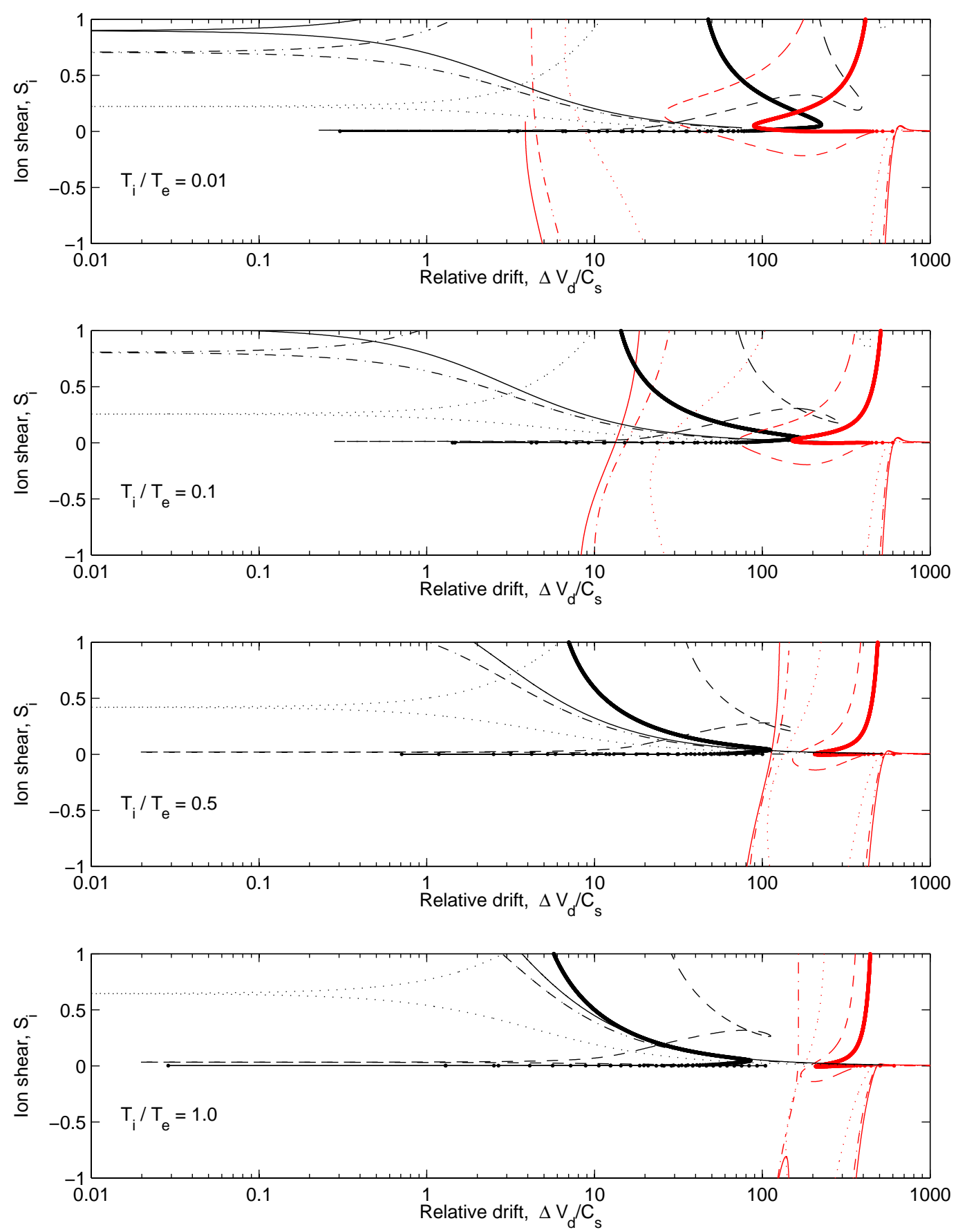

Fig. 3. Threshold conditions obtained using the kinetic dispersion relation Eq. (1) for $T_{\mathrm{i}} / T_{\mathrm{e}}=0.01$ top panel, $T_{\mathrm{i}} / T_{\mathrm{e}}=0.1$ second panel, $T_{\mathrm{i}} / T_{\mathrm{e}}=0.5$ third panel and $T_{\mathrm{i}} / T_{\mathrm{e}}=1.0$ bottom panel. The red traces correspond to the upper branches solutions of the threshold conditions that are shown in Fig. 4. The line scheme is the same as in Fig. 1.

The results of our collision frequency ratio study are shown in Fig. 6 where the top panel shows how the threshold condition changes with shear $S_{\mathrm{i}}$ and the absolute value of the relative drift $\left|\Delta V_{\mathrm{d}} / C_{\mathrm{s}}\right|$ when $\tau=1.0, v_{\mathrm{i}} / \Omega_{\mathrm{i}}=0.01$ and $v_{\mathrm{e}} / \nu_{\mathrm{i}}=100$. We have used the same frequency range as in
Figs. 1-5. Likewise, in similar fashion to previous figures, the lower left panel of Fig. 6 shows how the absolute value of the relative drift changes with $\theta$ while the lower right panel shows how the absolute value of the shear changes as a function of aspect angle. 

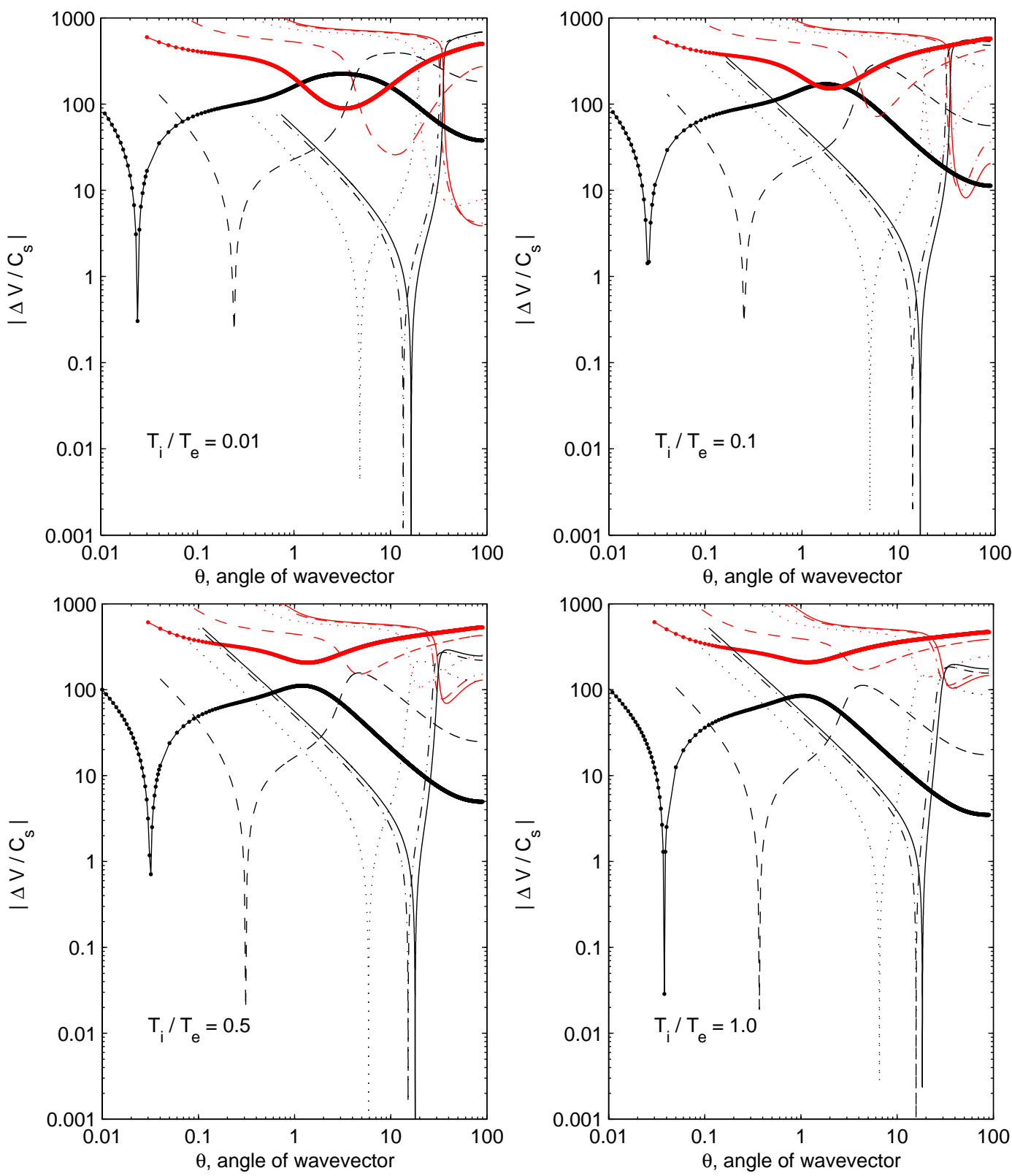

Fig. 4. Relative drift as a function of wavevector angle for the case described in Fig. 3. The angle is $0^{\circ}$ when the angle is perpendicular to $\mathbf{B}$ and $90^{\circ}$ when parallel to it. The line scheme is the same as in Fig. 1.

When we compare the top panel of Fig. 6 with the bottom panel of Fig. 3, we observe very little differences between the figures. The same thing can be said of the comparison between the lower left panel of Fig. 6 with the lower right panel of Fig. 4. The only differences are that when the value of $\nu_{\mathrm{e}} / \nu_{\mathrm{i}}$ is increased, the minima for $\omega_{\mathrm{R}} / k C_{\mathrm{s}}=0.02$ and 0.1 move towards the right or to larger aspect angles while the minima for $\omega_{\mathrm{R}} / k C_{\mathrm{s}}>0.1$ remain relatively stationary. For example, when $v_{\mathrm{e}} / v_{\mathrm{i}}=10.0$ and $\tau=1.0$ the minimum in the relative drift for $\omega_{\mathrm{R}}=0.02$ is located at $\theta \sim 0.03^{\circ}$ which corresponds to a shear of $S_{\mathrm{i}} \sim 0.002$. When $\nu_{\mathrm{e}} / \nu_{\mathrm{i}}$ is increased to 100.0 while keeping all the other parameters the same, the minimum in the relative drift for $\omega_{R}=0.02$ is located at $\theta \sim 0.1^{\circ}$ corresponding to a shear magnitude $\left|S_{\mathrm{i}}\right| \sim 0.005-0.006$. From these results we can conclude that changing $v_{\mathrm{e}} / v_{\mathrm{i}}$ by one order of magnitude from 10 to 100 does not significantly affect the solutions, at least for our choice of the other parameters.

Finally, in order to more clearly illustrate the impact of the collision frequency on the threshold conditions in the kinetic 

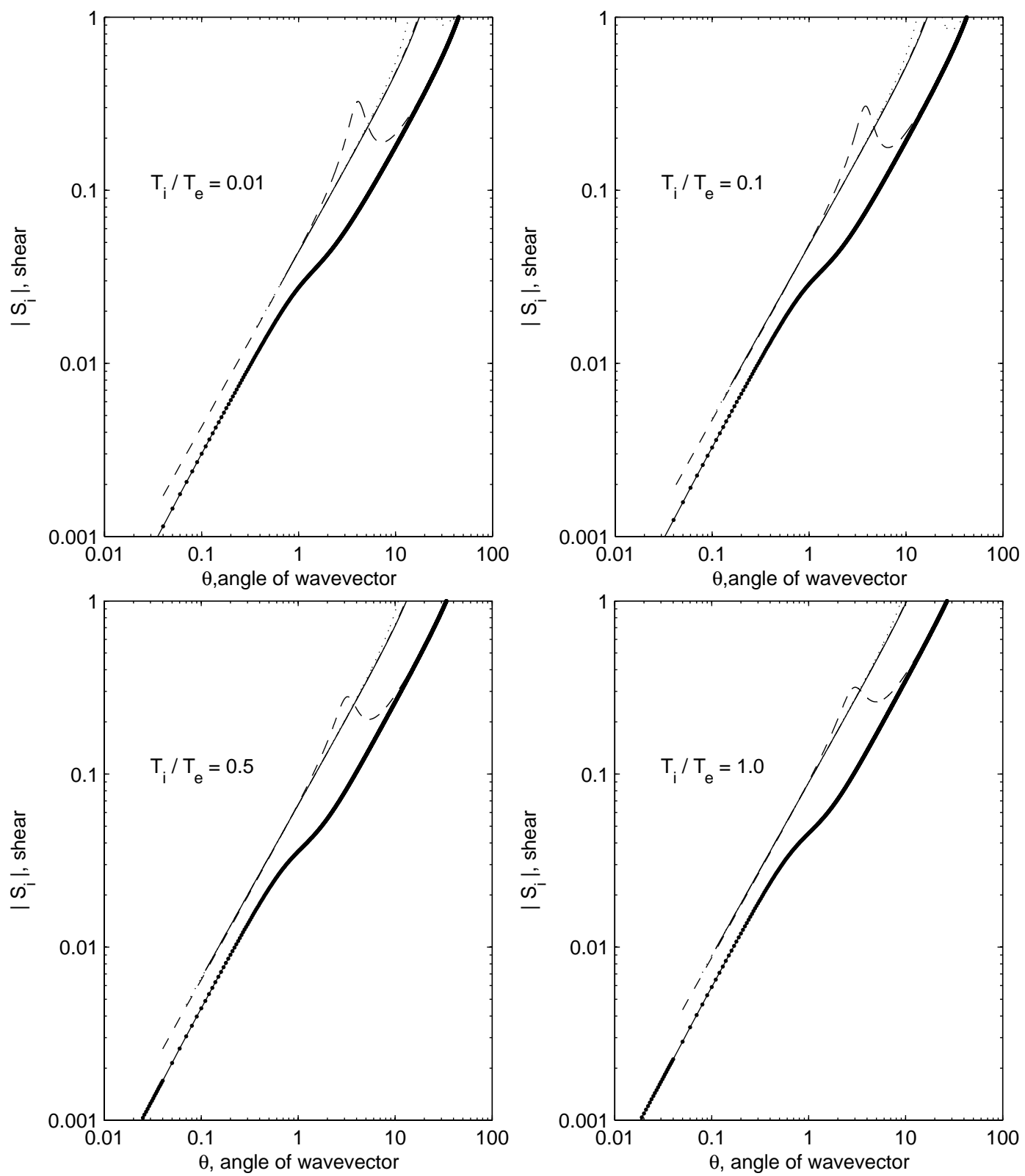

Fig. 5. Velocity shear as a function of wavevector angle for the case described in Fig. 3 . The angle is $0^{\circ}$ when the angle is perpendicular to B and $90^{\circ}$ when parallel to it. The line scheme is the same as in Fig. 1.

regime, we have also decreased the ion collision frequency by one order of magnitude for the case $\tau=0.1$, while maintaining all other variables the same. The results of the calculations are shown in Fig. 7, using the same format as in Fig. 6. Figure 7 confirms the trends that could be inferred already in the fluid limit, in the St.-Maurice et al. (2006) study. First of all, the small frequency ion shear driven instability modes associated with the "lower branch" solutions become more difficult to excite, in that they all require stronger to much stronger shears to be created under the constraint
$\left|\Delta V_{D}\right| \ll C_{s}$. This can be seen by the fact that in Fig. 7 we now see the $\omega_{R}=0.1 k C_{s}$ modes excited with weak relative drifts if the shears are of the order of 0.1 ; meanwhile, the higher frequency modes have not yet gone through the resonance when $S_{i}=1$. This contrasts with Fig. 3 where the required shears were much smaller at all frequencies. These results are consistent with the analysis presented by St.-Maurice et al. (2006) which showed that the shear modes can only be excited in the presence of collisions in the fluid regime. 

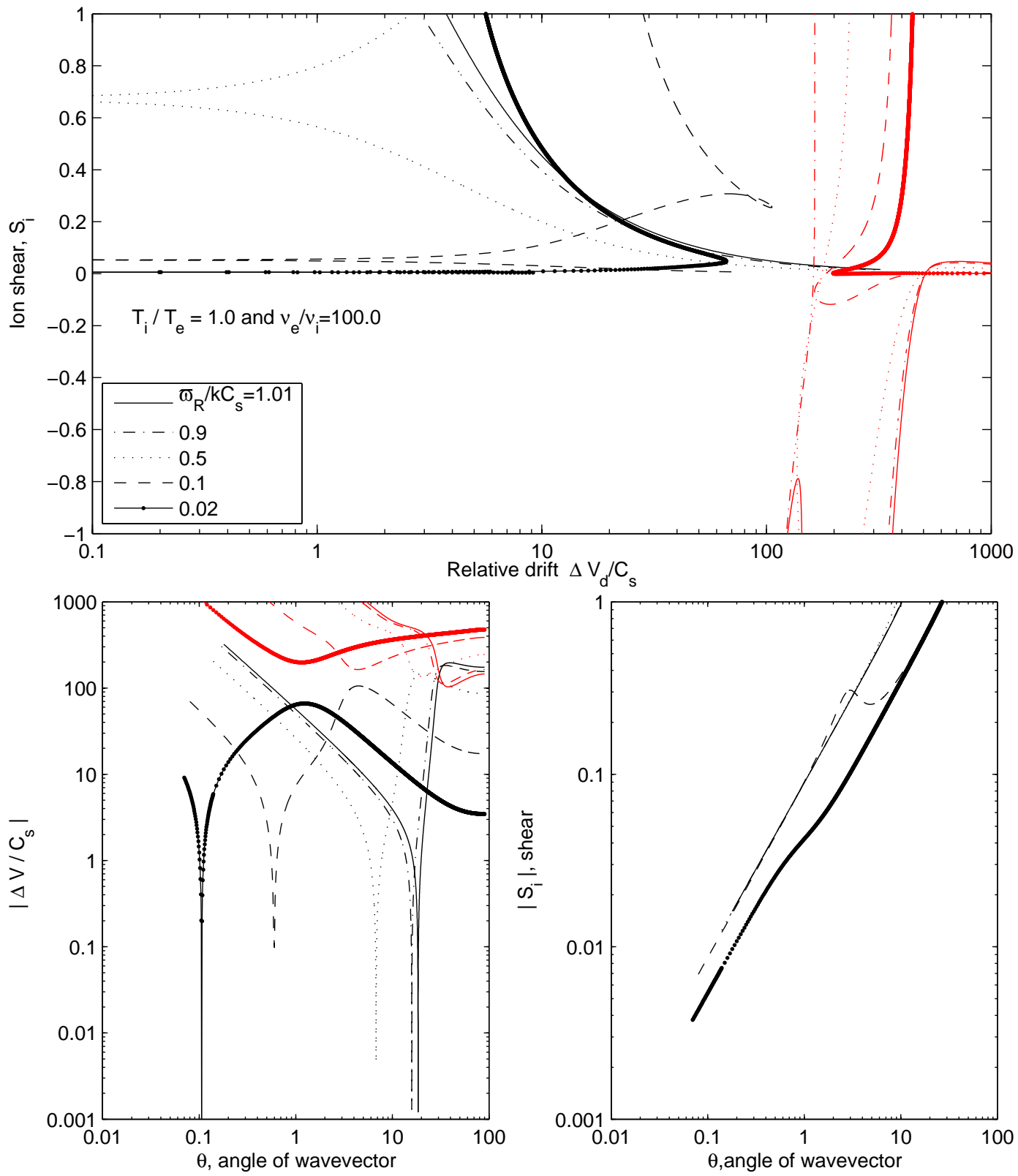

Fig. 6. Threshold conditions for $\nu_{\mathrm{e}} / \nu_{\mathrm{i}}=100.0$ and $\tau=1.0$ using the generalized kinetic dispersion relation. The top panel shows the shear $S_{\mathrm{i}}$ versus the relative drift $\Delta V_{\mathrm{d}} / C_{\mathrm{s}}$. The bottom left panel shows the relative drift as a function of angle. The bottom right panel shows the velocity shear as a function of angle. The line scheme is the same as in Fig. 1.

The second feature that we can notice from Fig. 7 is that, for a given value of $\omega_{R} / k C_{S}$ the "upper branch" modes are, by contrast to the small frequency ion shear driven modes, easier to excite in terms of $\left|\Delta V_{D}\right| / C_{s}$. This is particularly true for shears near zero or negative. Again, we stress that these results are entirely consistent with the analysis presented by St.-Maurice et al. (2006) who contrasted a zero collision frequency case to the case $v_{e} / v_{i}=10$ and $v_{i} / \Omega_{i}=0.01$ that we have also used here as our benchmark.

\section{Discussion and conclusions}

We have seen that, much like in the fluid limit considered by St.-Maurice et al. (2006), there are two classes of solutions: the first one is found at small frequencies, with nearly perpendicular wave-vectors that are very sensitive to shears (lower branch solutions). The second one deals with frequencies much closer to $k C_{\mathrm{s}}$, and the modes have more substantial deviations from perpendicularity, greater sensitivity to drifts 

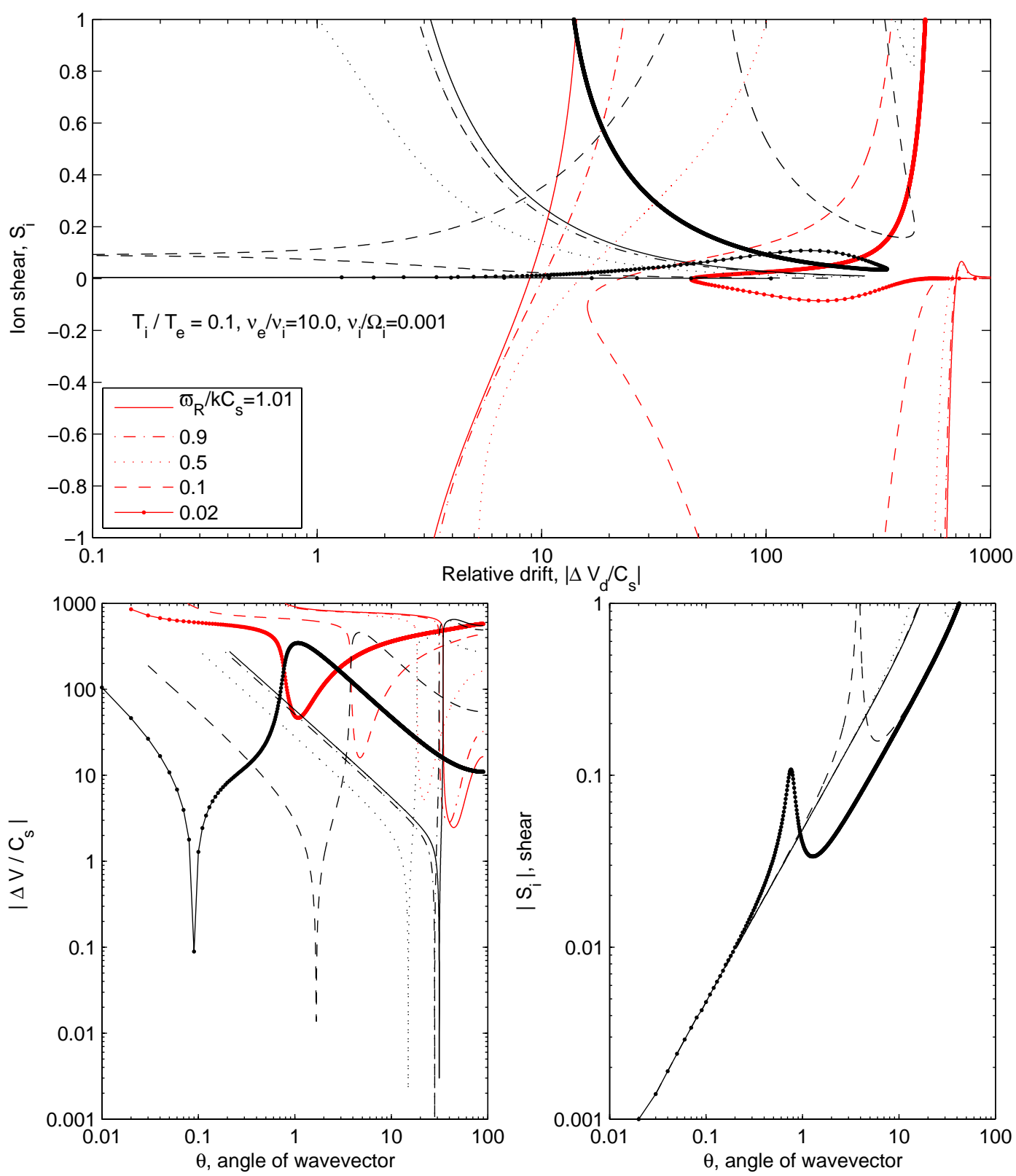

Fig. 7. Same as Fig. 6, but for $v_{\mathrm{i}} / \Omega_{\mathrm{i}}=0.001, v_{\mathrm{e}} / v_{\mathrm{i}}=10.0$ and $\tau=0.1$.

and less sensitivity to shears. Based on the analysis presented in St.-Maurice et al. (2006) it is clear that the first class is essentially the small frequency ion shear driven instability analyzed first by Basu and Coppi (1989). The second class is more closely related to the streaming instability discussed initially by Kindel and Kennel (1971) in that it is much less sensitive to shears than the first.

In the present work we have explored the kinetic solution to the dispersion relation, meaning that we were not limited to small $T_{\mathrm{i}} / T_{\mathrm{e}}$ ratios, among other things. We have found that the temperature ratio did not produce any surprise. Thus, just like for the fluid case, for $v_{\mathrm{i}} / \Omega_{\mathrm{i}}$ of order 0.01 , the easiest modes to excite still had low frequencies $\left(\omega_{\mathrm{R}} \ll k C_{\mathrm{s}}\right)$ and were within one degree of perpendicularity to the magnetic field. Such modes require relatively small current densities (relative drifts less than the ion acoustic speed) and shears $\left|S_{\mathrm{i}}\right|=\left|V_{\mathrm{di}}^{\prime} / \Omega_{\mathrm{i}}\right|$ that can be as small as $10^{-3}$. We have found that these modes did not depend strongly on the ratio $T_{\mathrm{i}} / T_{\mathrm{e}}$ or on the ratio $v_{\mathrm{e}} / v_{\mathrm{i}}$. They are nevertheless somewhat easier to excite with smaller values of $T_{\mathrm{i}} / T_{\mathrm{e}}$.

We have found that modes with relative drifts less than the ion-acoustic speed and frequencies of the order of $k C_{\mathrm{s}}$ can 
nevertheless be excited if the shears are increased. For what we have called the "lower branch" solutions, we have found that deviations from perpendicularity that could be as large as $10^{\circ}$ or more can happen in this case, provided the shear parameter $\left|S_{\mathrm{i}}\right|$ reaches a value of the order of 0.2 . These, however, are not the kinds of infinitesimal shears that were discussed in the collision-free work of Gavrishchaka et al. (1998), and such shears seem unlikely: for instance, with $1 \mathrm{~km} / \mathrm{s}$ peak ion drifts along the magnetic field and $\mathrm{O}^{+}$ions, $\left|S_{\mathrm{i}}\right|=0.2$ would imply a horizontal shear scale of the order of $20 \mathrm{~m}$. In view of various observations of ion outflows in what appear to be intense current regions (Kagan and St.-Maurice, $2005)$ and of the simulations performed by Noël et al. (2000, 2005), this scale would appear to be smaller than what might be possible by roughly one order of magnitude. However, if even relatively modest parallel current densities are allowed to flow, with $V_{\mathrm{de}}$ of the order of, say, $5 C_{\mathrm{s}}$ to $10 C_{\mathrm{s}}$, the requirement for the shears does go down, as seen in Figs. 1 and 3 and some of our other panels. We could therefore expect modes with frequencies of the order of $0.5 k C_{\mathrm{s}}$ and wavevectors at an angle $5^{\circ}$ to $10^{\circ}$ away from perpendicularity to the magnetic field to be excited in "type-2" ion outflow regions, where intense parallel currents also appear to be observed, this near the edges of auroral arcs.

The above may have practical applications to radar observations of irregularities in the vicinity of intense parallel current regions near the edge of arcs. However, we should note that the excited modes that we have discussed thus far are nearly perpendicular to the magnetic field and favor lower frequencies. The excited modes would therefore be seen as essentially field-aligned and drifting with the plasma. Radars in the SuperDARN network are particularly well suited to catch these kinds of echoes. Horizontally narrow regions of ion outflow might, for these radars, therefore be seen as localized regions of strong radar echoes in the vicinity of intense velocity shears (where parallel current densities and possibly type- 2 ion outflows are most likely to be found). Still, it has also been suggested that strong ion acoustic echoes occasionally observed with incoherent scatter radars along the magnetic field direction (or close to it) in the F-region are produced by localized intense parallel current densities (see Sedgemore-Schelthess and St.-Maurice, 2001, for a review). The interest of the present work is to see if in the presence of ion outflows and associated shears we could obtain a decrease in the threshold conditions for this kind of observation geometry. In our figures this geometry coincides to solutions with large angles $\theta$, all of which belong to the "upper branch" of our solutions. These solutions are, however, largely independent of the shears for our collisional solutions. As a specific example, consider the upper branch trace for $\omega_{\mathrm{R}}=k C_{\mathrm{s}}$ in Fig. 3 in the second panel from the top: the shears only play a minor role in changing the threshold value while Fig. 4 indicates that for this family of solutions the angles are all fairly closely aligned with the magnetic field direction. In other words, in our solutions, the factor that most clearly fa- cilitates the trigger of an instability is the temperature ratio $T_{\mathrm{i}} / T_{\mathrm{e}}$ : the lower this ratio is, the easier it is to trigger an instability. While there is nothing new to this result, we wish to simply emphasize here that shears do not play a significant role in lowering the threshold for this geometry, at least for the cases that we have studied here.

Finally, while we have focussed here on F-region applications, we should stress that our study should also be relevant to laboratory studies of shear-driven instabilities. In particular, Agrimson et al. (2001) demonstrated that shears clearly played a role in decreasing the threshold speed conditions in terms of $\left|\Delta V_{\mathrm{d}}\right|$. Although the authors did not give enough information about the collision frequencies in their system, we note that their shear parameter $S_{\mathrm{i}}$ was of order -1 . As seen by a comparison between the top panel of Fig. 7 and the second panel from the top in Fig. 3, the threshold speeds in the region $S_{\mathrm{i}} \rightarrow-1$ are rather sensitive to the collision frequency for what we've called here the "upper branch" modes. This stated, is should be clear from the comparison between Fig. 7 and Fig. 3 that for sufficiently small values of $v_{\mathrm{i}} / \Omega_{\mathrm{i}}$ the shears should indeed play a major role in significantly lowering the threshold speed.

In summary, while we can confirm that shears play an important role in lowering the threshold relative drift, collisions act to strongly favor the excitation of low frequency modes with wave-vectors very nearly perpendicular to the magnetic field. Collisions also strongly hinder the production by shears of modes with frequencies of the order of $k C_{\mathrm{s}}$. Such modes remain nevertheless possible in the ionosphere in view of the fact that strong ion outflow regions appear to coexist with regions of fairly intense parallel current densities at high latitudes. However, it would appear that the shears are relatively small so that these modes should be favoring wave-vectors that are rather close to perpendicularity to the magnetic field; at least for the calculations that we have carried out, the shears have very little impact on the threshold conditions of modes with wave-vectors closely aligned with the magnetic field direction for conditions that would be expected in the F-region ionosphere.

Acknowledgements. This work was supported in part by a Defence Research and Development Branch - Royal Military College fellowship, as well as research grants from the Natural Sciences and Engineering Research Council of Canada.

Topical Editor M. Pinnock thanks two anonymous referees for their help in evaluating this paper.

\section{References}

Agrimson, E., D’Angelo, N., and Merlino, R. L.: Excitation of IonAcoustic-Like Waves by Subcritical Currents in a Plasma Having Equal Electron and Ion Temperatures, Phys. Rev. Lett., 86, 52825285, 2001.

Basu, B. and Coppi, B.: Velocity shear and fluctuations in the auroral regions of the ionosphere., J. Geophys. Res., 94, 5316-5326, 1989. 
Berthelier, J.-J., Machard, C., Cerisier, J.-C., and Berthelier, A.: ULF electromagnetic turbulence in the high-latitude topside ionosphere, J. Geophys. Res., 93, 5701-5712, 1988.

Cerisier, J. C., Machard, C., and Pottelette, R.: MHD turbulence generated by time-varying field-aligned currents., J. Geophys. Res., 92, 11225-11230, 1987.

D'Angelo, N.: Kelvin-Helmholtz instability in a fully ionised plasma in a magnetic field, Phys. Fluids, 8, 1748-1750, 1965.

Earle, G. D., Kelley, M. C., and Ganguli, G.: Large velocity shears and associated electrostatic waves and turbulence in the auroral F-region., J. Geophys. Res., 94, 15321-15333, 1989.

Forme, F. R. E.: Parametric decay of beam-driven Langmuir wave and enhanced ion-acoustic fluctuations in the ionosphere: a weak turbulence approach, Ann. Geophys., 17, 1172-1181, 1999, http://www.ann-geophys.net/17/1172/1999/.

Ganguli, G., Keskinen, M. J., Romero, H., Heelis, R., Morre, T., and Pollock, C.: Coupling of microprocesses and macroprocesses due to velocity shear: an application to the low-altitude ionosphere, J. Geophys. Res., 99, 8873-8889, 1994.

Gavrishchaka, V. V., Ganguli, S. B., and Ganguli, G. I.: Origin of low-frequency oscillations in the ionosphere., Phys. Rev. Lett., 80, 728-731, 1998.

Gavrishchaka, V. V., Ganguli, S. B., and Ganguli, G. I.: Electrostatic oscillations due to filamentary structures in the magneticfield-aligned flow: The ion-acoustic branch, J. Geophys. Res., 104(A6), 12683-12693, 1999.

Kagan, L. M. and St.-Maurice, J.-P.: Origin of type-2 thermal-ion upflows in the auroral ionosphere, Ann. Geophys., 23, 13-24, 2005 , http://www.ann-geophys.net/23/13/2005/.

Kindel, J. M. and Kennel, C. F.: Topside current instabilities., J. Geophys. Res., 76, 3055-3078, 1971.

Liu, H. and Lu, G.: Velocity shear-related ion upflow in the lowaltitude ionosphere, Ann. Geophys., 22, 1149-1153, 2004, http://www.ann-geophys.net/22/1149/2004/.

Maggs, J. E. and Davis, T. N.: Measurements of the thicknesses of auroral structures., Planet. Space Sci., 16, 205-209, 1968.

Marklund, G., Sandahl, I., and Opgenoorth, H.: A study of the dynamics of a discrete auroral arc., Planet. Space Sci., 30, 179-197, 1982.

Noël, J.-M.: Small-scale electrodynamics in the auroral ionosphere, Ph d dissertation, The University of Western-Ontario, 1999.

Noël, J.-M. A., St.-Maurice, J.-P., and Blelly, P.-L.: Nonlinear model of short-scale electrodynamics in the auroral ionosphere, Ann. Geophys., 18, 1128-1144, 2000, http://www.ann-geophys.net/18/1128/2000/.
Noël, J.-M. A., St.-Maurice, J.-P., and Blelly, P.-L.: The effect of Eregion wave heating on electrodynamical structures, Ann. Geophys., 23, 2081-2094, 2005, http://www.ann-geophys.net/23/2081/2005/.

Ossakow, S. L. and Chaturvedi, P. K.: Current convective instability in the diffuse aurora, Geophys. Res. Lett., 6, 332-334, 1979.

Perron, P. J. G.: Shear and current driven electrostatic instability in a collisional ionosphere, Master's thesis, The Royal Military College of Canada, P.O. Box 17000 Station Forces, Kingston, ON K7K 7B4, 2004.

Rother, M., Schlegel, K., and Lühr, H.: CHAMP observation of intense kilometer-scale field-aligned currents, evidence for an ionospheric Alfvn resonator, Ann. Geophys., 25, 1603-1615, 2007 , http://www.ann-geophys.net/25/1603/2007/.

Schunk, R. W. and Nagy, A. F.: Ionospheres. Physics, plasma physics, and chemistry, Cambridge atmophseric and space science series, Cambridge University Press, Cambridge, United Kingdom, 2000.

Scime, E. E., Keesee, A. M., Spangler, R. S., Koepke, M. E., Teodorescu, C., and Reynolds, E. W.: Evidence for thermal anisotropy effects on shear modified ion acoustic instabilities, Phys. Plasmas, 9, 4399-4401, 2002.

Sedgemore-Schelthess, F. and St.-Maurice, J.-P.: Naturally enhanced ion-acoustic spectra and their interpretations, Surv. Geophys., 22, 55-92, 2001.

Spangler, R. S., Scime, E. E., and Ganguli, G. I.: Parallel inhomogeneous flows in a thermally anisotropic plasma: The electrostatic ion-acoustic branch, Phys. Plasmas, 9, 2526-2533, 2002.

St.-Maurice, J.-P., Noël, J.-M. A., and Perron, P. J. G.: An assessment of how a combination of shears, field-aligned currents and collisions affect F-region ionospheric instabilities, J. Plasma Phys., 72, Part 4, 1-20, doi:10.1017/s002237780600434x, 2006.

Trondsen, T. S. and Cogger, L. L.: High-resolution television observations of black aurora, J. Geophys. Res., 102, 363-378, 1997.

Wahlund, J. E., Forme, F. R. E., Opgenoorth, H. J., Persson, M. L. A., Mishin, E. V., and Volokitin, A.: Scattering of electromagnetic waves from a plasma: enhanced ion acousticfluctuations due to ion-ion two-stream instability., Geophys. Res. Lett., 19, 1919-1922, 1992. 